\title{
La prise en charge du tabou de la mort par les thanatopracteurs
}

Conséquences sur la santé et les conditions de travail

How embalmers handle the taboos surrounding death: consequences for their health and working conditions

\section{Marlène Biotteau et Anaïs Mayeur}

\section{OpenEdition}

Journals

Édition électronique

URL : http://journals.openedition.org/activites/2356

DOI : 10.4000/activites.2356

ISSN : 1765-2723

\section{Éditeur}

ARPACT - Association Recherches et Pratiques sur les ACTivités

\section{Référence électronique}

Marlène Biotteau et Anaïs Mayeur, « La prise en charge du tabou de la mort par les thanatopracteurs », Activités [En ligne], 7-1 | avril 2010, mis en ligne le 15 avril 2010, consulté le 30 avril 2019. URL: http://journals.openedition.org/activites/2356 ; DOI : 10.4000/activites.2356

\section{(@) $\odot \Theta \Theta$}

Activités est mis à disposition selon les termes de la licence Creative Commons Attribution - Pas d'Utilisation Commerciale - Pas de Modification 4.0 International. 


\title{
La prise en charge du tabou de la mort par les thanatopracteurs :
}

\section{conséquences sur la santé et les conditions de travail}

\section{Marlène Biotteau}

\author{
Consultante Ergonome \\ 133 rue de javel, 75015 Paris \\ marlene.biotteau@gmail.com
}

\author{
Anaiis Mayeur \\ Chercheure en Ergonomie \\ LIMSI-CNRS, Groupe CPU (Cognition, Perception Usage) \\ Bât 508 -BP 133 - 91403 ORSAY Cedex (F) \\ anais.mayeur@limsi.fr
}

\begin{abstract}
How embalmers handle the taboos surrounding death: consequences for their health and working conditions. This ergonomic study describes the invariants and variable work situations of embalmers. It examines the consequences of difficult work conditions and their effects on the practitioners' health. However the complexity of this profession, which must be apprehended for understanding and transforming work, requires a transdisciplinary approach to distinguish the various underlying levels of reality and logic. Even though society describes and regards embalming as degrading, it is nonetheless essential for the survival of society. This unwanted profession deals with "objects", i.e. corpses, that are regarded as "dirty" because they exemplify the taboos surrounding death. We will see how the profession may be regarded as "dirty work" from a societal perspective, resulting in a shortage of relevant legislation. These elements lead to social alienation and the pathogenic nature of the embalmers' working conditions.
\end{abstract}

KEYWORDS

Embalmers, taboo, "dirty work", legal context, health, working conditions

\section{1.- Introduction}

Une vague de chaleur, à l'été 2003, a emporté avec elle plus de 15000 morts en quelques jours (Prioux, 2006). Cette année-là, le nombre total de décès a été évalué à 550000 (Desesquelles, \& Richet-Mastain, 2004). Cette actualité, touchant de plein fouet l'opinion publique, a soulevé de nombreuses interrogations tant sur le plan de la mort et de ses circonstances, que sur le plan de la prévention, de la gestion, de l'organisation, et de l'information qui en fut faite (Alby, Benattar, Calvat, Caquet, Chapuisat, Charpentier, et al., 2004). Le relais médiatique a pointé des carences institutionnelles majeures dans la prise en charge des personnes âgées dans un contexte climatique exceptionnel. Cependant, ces préoccupations, certes primordiales, ont occulté un enjeu de fond: celui de la gestion et de la prise en charge d'une augmentation massive des décès. La catastrophe d'août 2003 peut, en effet, être en partie expliquée par l'absence de locaux adaptés aux températures excessives et durables, par un déficit de personnel dans le secteur de la gérontologie, par la lenteur administrative du secteur funéraire et l'insuffisance de structures mortuaires d'accueil (Aguilly, Batisse-Dauquaire, Bonello, Bremaud, Caquineau, Dupont, et al., 2004). Pour autant aucune réponse concrète n'a été apportée concernant la gestion et la prise en charge d'une augmentation massive des décès. D'autre 
part, les prévisions démographiques laissent entrevoir un accroissement des décès dans les années à venir, sans compter sur des résurgences de phénomènes climatiques. D'après les projections de l'INSEE, en 2050 en France, 69 habitants sur 100 seraient âgés de 60 ans ou plus, soit deux fois plus qu'en 2005 (Robert-Bobée, 2006). Les plus de 65 ans d'aujourd'hui, (16,5\% de la population, soit près de 10,6 millions d'individus), décéderont, au vue de l'espérance de vie (estimée à 77 ans pour les hommes et à 84 ans pour les femmes), dans les 10 à 20 prochaines années; génération suivie de près par celle du baby-boom ( $35 \%$ de la population française estimée à 64,3 millions d'habitants') (Pla, 2008). Autant de chiffres qui justifient l'importance d'étudier un secteur funéraire peu médiatisé et relégué aux rubriques nécrologiques.

La polémique suscitée révèle une méconnaissance des intervenants du secteur funéraire. Les nombreux professionnels impliqués dans la prise en charge de la mort ont été au centre du mois d'août 2003: de ce qu'ils nomment eux-mêmes l'enfer, d'une hécatombe révélatrice de leur difficulté à exercer leur métier, de leurs conditions de travail et de leur impuissance face à un système vieillissant et inadapté aux nouvelles donnes de cette société. Nous verrons dans quelle mesure les thanatopracteurs peuvent être considérés comme une "profession fantôme », peu reconnue par les institutions publiques et pourtant indispensable à la prise en charge des défunts. L'approche transdisciplinaire de cette recherche se distingue dans la mesure où elle déborde la discipline de l'ergonomie et souhaiterait viser une contribution au-delà de la recherche disciplinaire.

\section{1.- La thanatopraxie et les soins de conservation}

La thanatopraxie (du grec thanatos: génie de la mort et praxere: manipuler, traiter) est un ensemble de moyens (chimiques) et de techniques (médicales, chirurgicales) mis en œuvre, par un thanatopracteur, pour la conservation du corps des personnes décédées (Mayer, 2000). La forme moderne, telle que nous la connaissons aujourd'hui, est apparue en France en 1963. Peu de données sont disponibles, mais les professionnels de la thanatopraxie estiment qu'il y aurait actuellement entre 350 et 450 thanatopracteurs praticiens sur le territoire français, avec 1015 diplômés depuis 41 ans (estimation 2005). Les soins de conservation rentrent dans le cadre des services extérieurs des pompes funèbres, au même titre que la fourniture des cercueils ou des corbillards (Cf. article L. 2573-10 du code général des collectivités territoriales, Collectif, 2003).

L'appartenance aux services extérieurs et le statut de sous-traitants des pompes funèbres résultent d'un contexte historique. En France, au début des années soixante, trois français du secteur funéraire introduisirent cette pratique anglo-saxonne pour en faire une spécificité et un service des pompes funèbres. Le métier de thanatopracteur consiste à « traiter » la mort, opposément aux pompes funèbres qui s'occupent de la relation de service (contrat obsèques, etc.). Cela positionne ainsi la thanatopraxie en « aval de l'aval ». Par ailleurs, cette profession ne peut se rattacher moralement, symboliquement et légalement aux services de soins aux personnes vivantes. Il s'agirait d'une comparaison ad hoc entre professions paramédicales et/ou médicales, soignant le vivant; et les métiers du funéraires, gérants les morts, et plus précisément leur corps.

Les soins de conservation actuels répondent à plusieurs buts (Guibe-Hubert, 2002) : 1'hygiène, la préservation et la présentation des personnes décédées en vue de l'exposition. Le travail de préservation vise l'amélioration de l'aspect du défunt en permettant de retarder les effets de la thanatomorphose et de la transformation du corps suite au décès, par l'injection de 4 à 6 litres d'un liquide antiseptique et d'une ponction des liquides physiologiques. Des soins de conservation sur un défunt peuvent être à l'origine d'une demande de la famille ou de l'exécution d'un contrat obsèques. Il existe cependant des obligations législatives de faire effectuer des soins de conservation, et ce, malgré la volonté ou le culte de la famille ou du défunt (interdiction dans les confessions juives et musulmanes). A contrario, les soins sont proscrits lors d'un décès avec obstacle médico-légal, d'un accident du travail, d'une maladie professionnelle ou d'une atteinte de certaines affections (ex : infection à VIH).

1. Au 1er janvier 2009 


\section{2.- La perception occidentale de la mort et du cadavre}

Autrefois, dans les sociétés primitives, antiques et judéo-chrétiennes, des catégories de prêtres étaient exclusivement habilitées à s'occuper des défunts et à "gérer» la mort. Les tout premiers rituels datent des Néandertaliens, vers - 80000 : les défunts étaient ensevelis dans des fosses avec des rations de viande, des objets en silex et un crâne de bison (Frémy, \& Frémy, 2003).

Ces comportements primitifs continuent d'exister, mais sous une forme pondérée par la culture et l'intellect modernes (Mayer, 2000) et ce, en ayant connu une direction spécifique en Occident: la systématisation et le caractère méthodique des représentations humaines (Weber, 2006). Dans la religion catholique, la mort ne représente pas une fin en soi, mais correspond à un passage vers l'audelà. Anciennement, la toilette mortuaire était pratiquée sur chaque défunt, lequel était déshabillé et lavé. Les familles vivaient la mort comme un spectacle, qui n'était pas à la source d'une perversion, mais qui, au contraire, avait pour tâche de rappeler à l'Homme sa place dans l'univers. Faire une toilette mortuaire était, avant tout, un devoir permettant de rendre un dernier hommage au défunt et de sacraliser le corps en prévision de la Résurrection ${ }^{2}$. Aujourd'hui, les attitudes de déni et d'évitement face à la mort sont prééminentes. Nos sociétés tendent à donner une place privilégiée et exponentielle à des valeurs de jeunesse, de beauté, de santé et parallèlement à dévaluer la vieillesse, la disgrâce et la mort (Mayer, 2000). "L'homme achoppe toujours sur l'incurabilité de la mort (Thomas, 1980). Consécutivement, la valeur du corps humain est souvent dépréciée, parce qu'un cadavre symbolise ce que la société occidentale exècre le plus - la mort - précisément ce que notre culture tente d'effacer (Mayer, 2000). Finalement, le sacré (religieux) a disparu, mais bien qu'il ait été évacué, la réalité de la mort persiste. Ces figures de prêtres ont été remplacées par la science et la pratique médicale (Thomas, 2003); accompagnant ainsi la nouvelle représentation du cadavre, synonyme de danger et de contamination et justifiant les pratiques hygiénistes actuelles. Dans cette transition des mentalités, une substitution, un glissement, est en réalité survenu (Ariès, 1975).

Ce processus au cours des millénaires de la civilisation occidentale et, plus généralement, ce «progrès » auquel participe la science comme élément et comme moteur, ont une signification qui dépasse ces pures pratiques et techniques. En effet, le monde occidental se caractérise par une rationalisation orientée vers l'action pratique dans le monde, c'est-à-dire par une volonté de contrôle et de domination systématique de la nature et des hommes (Weber, 2006). La rationalisation développe les finalités, où buts et moyens sont sélectionnés en fonction de leur seule efficacité et non de leur contenu moral. Elle tend à rendre les relations sociales à la fois impersonnelles, instrumentales et utilitaires: dans leurs relations, les acteurs ne se considèrent que comme des moyens impersonnels, dans la poursuite de fins (Weber, Op. Cit.).

Avec la déchristianisation amorcée depuis les années 1960 (Ariès, 1975) et le déficit de sens entraîné qui n'a pas été remplacé (Gauchet, 1985), le corps est devenu tabou, frappé d'interdit. La mort, à défaut d'être conçue comme la fin d'une existence, d'une pensée, d'une histoire, est représentée typiquement comme une maladie incurable (Bacqué, 2002). La plupart des décès ont lieu en milieu hospitalier (en 2008, 543500 décès sont répertoriés en France et près de $70 \%$ des décès ont lieu, selon l'INSERM, dans un établissement de soins ou d'hébergement médicalisé) reléguant la prise en charge des défunts aux personnels soignants (Aguilly, et al., 2004, Thomas, 2003).

\section{3.- Le mépris du corps et de la thanatopraxie}

Rizet (1998) définit le mépris comme un sentiment et une attitude envers quelqu'un, un groupe, une chose, considérée comme indigne d'estime. Il positionne celui qui en use au-dessus de ce qu'il méprise : « Domination, mise à l'écart et condamnation sont les maîtres mots du mépris ». Selon cet auteur, le pape Innocent III élèvera le mépris au rang d'attitude morale et gage de salut, en opposant

2. Les chrétiens prétendent que Jésus est monté aux cieux et que par conséquent son corps n'a subi ni les affres du temps ni de la pourriture. Il a échappé à la Corruption: à savoir la décomposition, châtiment divin (Anonyme, 1992). 
le monde temporel (tel que le corps et les choses corporelles) au monde spirituel (âme, esprit). Cette conception met au banc l'intelligence pratique, du corps et de l'ingéniosité (tradition de la mètis grecque) au profit de l'intelligence conceptuelle.

Le mépris dans la société serait modelé par celui qui s'exprime dans le travail (Rizet, 1998). Le cadre du travail, dans lequel l'activité humaine engage de multiples ressorts de la créativité (stratégies de travail, catachrèses, etc.), est un enjeu majeur de l'observation du mépris dans les rapports humains. La division du travail a imposé une distinction rigide entre les activités intellectuelles et les activités sensorimotrices, principalement manuelles. Les premières, les professions intellectuelles, sont jugées supérieures aux autres. Rompre cette catégorisation n'est pas chose aisée (Poitou, 1992), car « les Hommes qui, dans leur métier, conçoivent plus qu'ils n'agissent, éprouvent un mépris non conscient (la plupart du temps) envers ceux dont la profession touche au corps ou utilise le corps » (Rizet, 1998).

Dans les professions peuvent être distingué un ensemble d'activités considérées moins honorables ou « sale boulot » (dirty works), que la société chercherait à déléguer afin d'accroître son honorabilité (Hughes, 1996). Les « dirty works » se définissent comme des actes, nécessaires à la survivance d'une société, mais qui restent désagréables, voire dégradants (Hughes, op. cit.), et dont l'activité, souhaitée par personne, consiste à prendre en charge des objets « sales » (Dant, \& Bowles, 2003). Au-delà de ce contact «souillé », ils renvoient à des comportements considérés comme impurs et choquants (Douglas, 1984). Selon la part de « sale boulot », il devient possible de distinguer et de hiérarchiser, dans un secteur donné, professions et métiers. La division morale du travail sépare toujours ceux, souhaitant prendre en charge le sale boulot, des « hommes bien » (Hughes, 1996).

L'acquisition d'une identité et l'incorporation de valeurs liées au métier (ou à l'activité à exercer) qui se construisent au cours des rapports sociaux, dans le milieu professionnel existent tant que la perception de l'activité (le réel) entre l'individu et la société concorde et que cette relation entre «moi » et les « autres » est possible (Sigaut, 1991). Les individus rencontrant des expériences négatives du mépris, s'affirment dans une lutte pour la reconnaissance de différentes formes d'identités lésées (Honneth, 1996). Cette lutte témoigne de l'altération de certains liens nécessaires à la construction identitaire, et de la genèse d'un processus d'aliénation (mentale, culturelle ou sociale). Dans l'aliénation sociale, le sujet maintien un lien avec le réel, le travail, mais n'est pas reconnu par autrui. Il est isolé et dans une situation psychologique délicate où personne ne lui permet de savoir si ce lien qu'il entretient avec le réel est fondé ou légitime (Jean, 2006).

\section{4.- La profession de thanatopracteur et son contexte juridique}

Dans le cadre de cette recherche, ont été répertoriés et croisés les textes juridiques relatifs à des éléments ou à la profession elle-même des thanatopracteurs. Depuis 1994, les thanatopracteurs sont titulaires d'un diplôme national (Cf. article L. 2223-45 du code général des collectivités territoriales, Collectif, 2003) et disposent d'une capacité professionnelle après avoir suivi une formation théorique et pratique auprès d'instituts et de thanatopracteurs habilités (Monsembernard, \& Vidal, 2003, Cf. articles D.2223-37, 49, 122 et 126 du code générale des collectivités territoriales, Collectif, 2003 et J.O. n 143 du 23 juin 1998, p. 9502). Toutefois, actuellement, aucune carte professionnelle ou document officiel ne permet de justifier leur habilitation.

Les thanatopracteurs exercent de façon ambulatoire, se déplacent et interviennent dans les chambres funéraires, les maisons de retraite, les chambres mortuaires, les instituts médico-légaux et au domicile des particuliers (Monsembernard, \& Vidal, 2003). Cependant, seuls les funérariums doivent fournir des dispositions matérielles particulières aux thanatopracteurs (ex: table de préparation, dispositif de désinfection des instruments, ventilation). Pourtant, quatre risques (toxicologique, biologique, infectieux et de pathologie rachidienne) sont répertoriés dans les situations de travail des thanatopracteurs. Par exemple, le formaldéhyde, fluide de conservation manipulé en permanence, irritant pour les yeux et les voies aériennes supérieures, est un allergène puissant cutané et respira- 
toire, responsable d'eczéma, d'urticaire ou d'asthme. Il appartient au groupe 2A de la classification du CIRC, à savoir un cancérogène probable pour l'homme (cancers rhino-pharyngés). De plus, est soumise à une législation très stricte (Collectif, 2004) la production de déchets d'activité de soins à risques infectieux (D.A.S.R.I.) liés aux matériels piquants, coupants, aux produits sanguins et/ou aux déchets anatomiques humains (Charbotel, Forissier, Hours, \& Bergeret, 2003). Ces déchets sont générés notamment dans la pratique de la thanatopraxie. Ainsi, la législation qui encadre et définit les D.A.S.R.I. octroierait indirectement les équipements et conditions d'hygiène spécifiques au cadre de travail des thanatopracteurs. Dans cette recherche, ces conditions d'exercice pour les praticiens ne sont apparues que très partiellement recouvertes ( $\mathrm{cf}$. résultats pages suivantes).

L'encadrement juridique parcellaire de la thanatopraxie interroge l'intégrité de cette profession. Tout collectif qui s'adonne à une tâche peut être considéré, de la part de l'Etat, comme ayant reçu la mission de l'accomplir. L'exercice de cette activité conduit le groupe à souhaiter se stabiliser en obtenant une autorisation légale d'exercer, validée par un concours (licence en anglais), qui permet de limiter la concurrence. Les dépositaires de cette permission octroyée acquièrent une mission reconnue et une responsabilité collective (mandate en anglais) qui valorise le groupe et définit le sens social de l'activité. Le groupe devient ainsi une profession (Hughes, 1996) dont la caractéristique décisive, et la plus précieuse est « une autonomie reconnue délibérément qui comprend le droit exclusif de décider qui est autorisé à accomplir le travail et comment celui-ci doit être » (Freidson, 1971). À l'image de la médecine, qui s'est constituée comme un champ scientifique et de connaissances objectives, et non plus hypothétiques et comparatives, à partir de la possibilité légale d'effectuer des vivisections sur des corps humains vivants (Rizet, 1998).

Forts de la conviction d'avoir une mission qu'ils seraient seuls à pouvoir accomplir, les « licenciés » entameraient une action de confiscation monopolistique (Hughes, 1996), dont les chances de réussite seraient d'autant plus grandes qu'ils parviendraient (i) à accomplir des tâches qui mettent en danger le reste de leurs congénères, ce qui est le cas des thanatopracteurs via la conservation des corps de défunts; (ii) et à faire preuve d'une force morale rare du fait de la gestion de « situations de culpabilités », pour lesquels le monde extérieur a des réponses passionnées, traditionnelles ou pour ce qui concerne les thanatopracteurs représente un tabou sociétal. Cela est à rapprocher de Freidson (1971) pour lequel une perte d'autonomie d'une profession consiste en la dislocation d'un monopole, la diminution du contrôle et la disparition d'un consensus symbolique.

\section{2.- Objectifs de l'étude}

Nous avons vu, tout d'abord, le rôle et la responsabilité sociale et sociétale des thanatopracteurs au regard de l'évolution démographique et des obligations législatives, leur statut de sous-traitant non monopolistique des pompes funèbres et la particularité de leur profession, outre l'activité même, dans une intervention en «aval de l'aval ». Puis a été présentés, la transition des mentalités sur la mort et le cadavre humain qui tend vers un hygiénisme, une rationalisation, mais aussi une tabouisation; le mépris relatif aux activités manuelles, liées au corps, et de «sale boulot », pour lesquelles s'engage une lutte pour la reconnaissance. Enfin, nous avons abordé l'encadrement juridique, au sens de Hughes avec les notions de mandate et de licence, mais aussi encadrement parcellaire dans l'absence de « la confiscation monopolistique » entraînant pour les praticiens: perte de consensus symbolique, d'autonomie et de contrôle. Dans cette description de cette profession, comment ne pas considérer face à un cadre et à des conditions de travail aussi aléatoires, les difficultés du travail réel et du réel du travail? Leurs conséquences en terme d'atteintes à la santé (physique, psychique, mentale et sociale) ne serait-ce qu'au regard des risques infectieux ou d'une aliénation sociale? 


\section{3.- Méthodologie}

Les thanatopracteurs ont un statut de salariés des pompes funèbres ou de marbreries/fleuristes; celui d'indépendant, s'instaure peu. 4 entreprises de l'Ile-de-France ont participé pendant 6 mois à cette recherche. Les professionnels ont accepté d'être systématiquement accompagnés dans leur activité. Face à la pénurie de données relatives à la thanatopraxie, l'actualité et l'étude des documents législatifs décrits ci-dessus ont permis de dessiner une première ébauche de l'encadrement et des conditions de travail du métier des thanatopracteurs. Cependant, ce corpus juridique, bien entendu, insuffisant pour décrire le prescrit du travail, nous a conduit à élaborer une première méthode d'analyse (phase 1), qui se fonde sur un ensemble d'observations, une analyse de l'activité et permet d'extraire les invariants de cette profession. Puis, afin d'étudier et de comparer les aspects réels et prescrits, une analyse de la variabilité des situations de travail des thanatopracteurs s'appuie sur une double méthodologie: tout d'abord, l'élaboration et la passation de questionnaires (phase 2), puis l'élaboration et la passation d'entretiens semi-directifs (phase 3) (cf. récapitulatif de la méthodologie, tableau 2). Cette méthodologie permet d'envisager dans quelle mesure un cadre de travail peu défini, dans la plupart des lieux d'intervention, et ce, malgré une activité sui generis, concourrait aux difficultés relatives au cadre de travail et à l'émergence de risques pour la santé de ces praticiens.

\section{1.- Phase 1 : analyse de l'activité et des invariants des conditions de travail par 37 observations}

Cette phase d'observation (37 journées d'observations) réalisée dans tous les lieux d'intervention à l'exception des I.M.L. ont permis via une analyse de l'activité, d'une part, de décrire les tâches (invariants) des praticiens (cf. partie 4.1., tableau 3) à effectuer (ex: les techniques employées, les instruments utilisés, la chronologie des soins, le jargon professionnel, les équipements et l'état des locaux) et d'autre part, d'élaborer l'instrument de recueil de données de la phase 2, ainsi que la grille d'entretien de la phase 3 .

\section{2.- Phase 2: analyse de la variabilité par 100 questionnaires dits « fiches d'intervention »}

Étant donné les caractéristiques ambulatoire et aléatoire de la thanatopraxie, les fiches d'interventions (cf. figure 1), construites sur la base de la phase 1, avaient comme premier objectif de permettre des observations que l'on pourrait qualifier de « différées » sur un plan temporel, mais systématisées et conservant la nature des composantes et des conditions de travail dans lesquelles se déroulaient les soins. Chaque fiche contient les données relatives à un soin effectué. Ainsi 100 rendus de soins ont pu être étudiés. Pour ce faire, les fiches se composent de deux parties distinctes. Les fiches, qui se présentent sous un format de poche, comportent une consigne générale: "cochez les cases en lien avec votre situation lors de l'intervention ». Une première partie, le descriptif, informe sur le jour, la période de la journée, le département, le lieu, et le type d'intervention des soins pratiqués; une seconde partie, problèmes rencontrés, décrit les aspects relationnels, équipements et état des locaux, problèmes divers. Dans son second objectif, ces fiches permettaient d'investiguer la pénibilité constatée par les praticiens au cours des soins. En effet, une troisième partie, l'évaluation des conditions de travail, se compose d'une échelle graduée de 1 (= conditions de travail excellentes) à 6 (= conditions de travail très pénibles). Les praticiens situaient à la fin de chaque soin, le niveau ou le degré de pénibilité ressentie. Les tableaux de contingence (test du c2), calculant les coefficients de corrélation, ont été retenus lorsque les seuils de significativité étaient inférieurs ou égaux à la valeur $\mathrm{a}=0,05 ;\left(\mathrm{p}(\mathrm{Chi} 2) \leq .05\right.$.). Des résultats ${ }^{3}$ sont reportés tout au long de la partie 4.3.

3. Utilisation du logiciel Statview 4.5. 


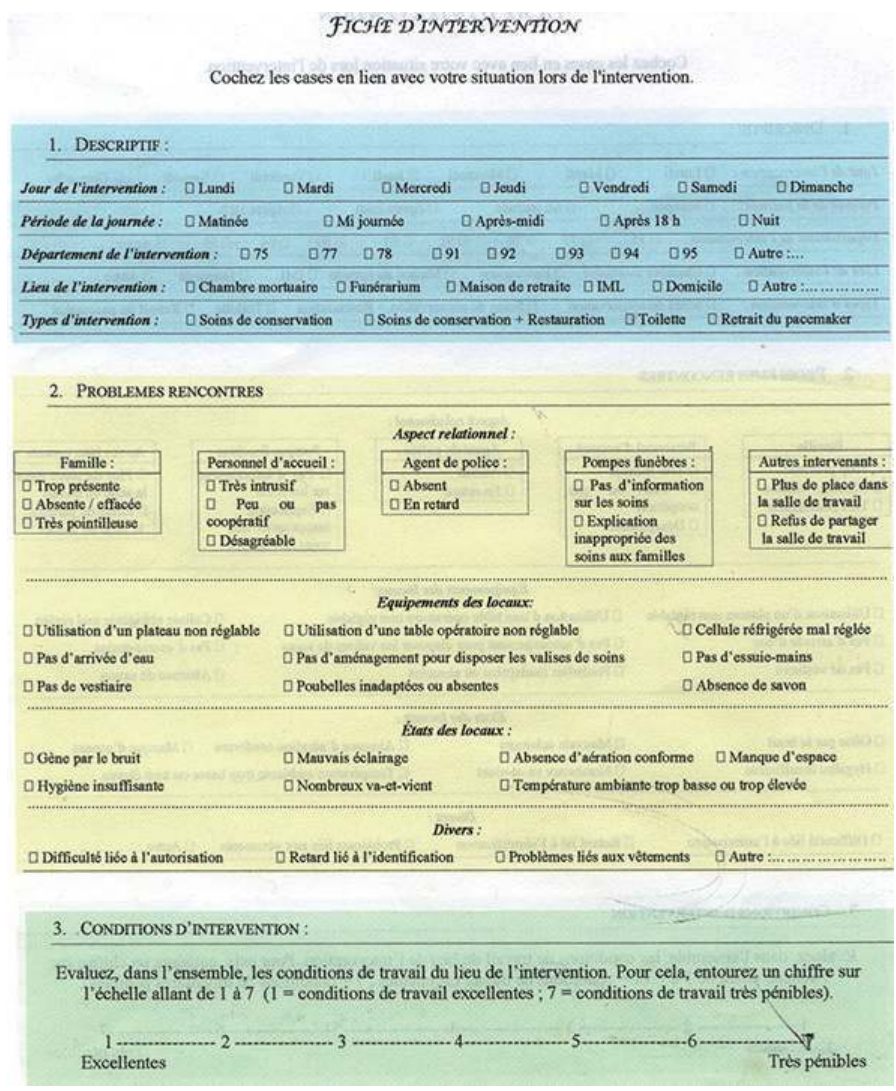

Figure 1: Exemple d'une fiche d'intervention

Figure 1: Example of a task sheet.

\section{3- Phase 3 : analyse de la variabilité à travers la passation de huit entretiens semi-directifs}

Un dernier niveau d'investigation, issu de la phase 1 et établi à travers des entretiens semi-directifs, a permis, d'une part, d'approfondir les situations relevées dans les fiches d'intervention (ex : précision de certaines situations de travail et des stratégies de travail mises en places par les opérateurs) et, d'autre part, de questionner les hypothèses d'une atteinte de la santé et des conséquences sur l'identité et les pairs.

1-Dans les établissements (à l'exception des domiciles), disposez-vous d'une salle de soins (ou de thanatopraxie) destinée à votre activité ?

2-Lorsque ces locaux ne sont pas adaptés et qu'il y a une salle d'autopsie, y avez-vous accès?

3-En cas de refus d'accès à une salle d'autopsie ou qu'il n'existe pas de salle tout simplement, travaillezvous dans la salle des cellules réfrigérées?

4-Y a-t-il des salles de cellules réfrigérées adaptées à votre activité?

5-Avez-vous déjà travaillé dans un lieu inadapté tel qu'un couloir, un cagibi ou autre bizarrerie?

6-Les conditions d'intervention dans un domicile sont très différentes, avez-vous déjà pratiqué un soin par terre? Dans un cagibi? Dans une caravane?

7-Les locaux se situent-ils toujours dans les niveaux inférieurs des établissements?

8-Les systèmes d'aération sont-ils au nombre de deux? Pour aérer, lors d'absence ou de dysfonctionnement d'un système d'aération, les locaux sont-ils équipés de fenêtres? Si ce n'est pas le cas, devez-vous ouvrir les portes de la salle?

Tableau 1: Extrait de la grille d'entretien.

Table 1: Extract from a interview grid. 
Des questions annexes ont porté sur les caractéristiques individuelles, l'organisation et temps de travail et des facteurs extra-professionnels. Pour ce faire, une grille d'entretien (cf. tableau 1) a été au préalable constituée. Les entretiens ont été codés à la suite d'une analyse de contenu de type thématique. Du contenu manifeste, des thèmes ont été dégagés, via un découpage des réponses apportées aux questions et une classification de ces derniers, en catégorie. Les tableaux de contingence (test du c2), calculant les coefficients de corrélation, ont été retenus lorsque les seuils de significativité étaient inférieurs ou égaux à la valeur $\mathrm{a}=0,05 ;(\mathrm{p}(\mathrm{Chi} 2) \leq .05$.). Des résultats sont reportés dans la partie 4.2.

\begin{tabular}{|c|c|c|c|c|c|}
\hline & Phases & Buts et intérêts & Population & Remarques & Résultats \\
\hline $\begin{array}{l}\mathrm{P} \\
\mathrm{R} \\
\mathrm{E} \\
\mathrm{S} \\
\mathrm{C} \\
\mathrm{R} \\
\mathrm{I} \\
\mathrm{T}\end{array}$ & $\begin{array}{l}\text { Phase 1: } \\
\text { Analyse de } \\
\text { l'activité } \\
37 \text { observations } \\
\text { systématisées }\end{array}$ & $\begin{array}{l}\text { - Décrire le plus } \\
\text { finement possible } \\
\text { l'activité des } \\
\text { thanatopracteurs } \\
\text { - Permettre la } \\
\text { mise en place } \\
\text { d'instruments de } \\
\text { recueils de données } \\
\text { les mieux adaptés }\end{array}$ & $\begin{array}{l}6 \text { praticiens ( } 2 \\
\text { femmes et } 4 \\
\text { hommes), dans } \\
\text { tous les lieux } \\
\text { d'exercice de la } \\
\text { thanatopraxie, à } \\
\text { l'exception des } \\
\text { I.M.L. }\end{array}$ & $\begin{array}{l}\text { - } 15 \\
\text { observations } \\
\text { dans des } \\
\text { chambres } \\
\text { funéraires } \\
-18 \text { dans des } \\
\text { chambres } \\
\text { mortuaires } \\
-3 \text { dans des } \\
\text { maisons de } \\
\text { retraite } \\
\text { - } 1 \text { à domicile }\end{array}$ & $\begin{array}{l}\text { Se référer à la } \\
\text { page } 102 \text { pour } \\
\text { les invariants }\end{array}$ \\
\hline
\end{tabular}

En a découlé les questionnaires et les entretiens :

\begin{tabular}{|c|c|c|c|c|c|}
\hline \multirow[b]{2}{*}{$\begin{array}{l}\mathrm{R} \\
\mathrm{E} \\
\mathrm{E} \\
\mathrm{L}\end{array}$} & $\begin{array}{l}\text { Phase 2: } \\
\text { Questionnaire } \\
100 \text { fiches } \\
\text { d'interventions }\end{array}$ & $\begin{array}{l}\text { Étudier la pénibilité } \\
\text { des conditions } \\
\text { de travail des } \\
\text { thanatopracteurs }\end{array}$ & $\begin{array}{l}13 \text { praticiens } \\
\text { hommes ( } 12 \text { ont } \\
\text { rempli } 8 \text { fiches } \\
\text { et } 1,4 \text { fiches) }\end{array}$ & $\begin{array}{l}100 \text { fiches } \\
\text { cotées pour } \\
\text { les analyses } \\
\text { statistiques }\end{array}$ & $\begin{array}{l}\text { Se référer aux } \\
\text { pages } 104 \text { à } 107\end{array}$ \\
\hline & $\begin{array}{l}\text { Phase } 3: \\
8 \text { entretiens } \\
\text { semi-directifs }\end{array}$ & $\begin{array}{l}\text { - Aborder les } \\
\text { questions laissées } \\
\text { en suspens dans les } \\
\text { fiches d'intervention } \\
\text { - Répondre aux } \\
\text { hypothèses d'une } \\
\text { atteinte de la santé } \\
\text { et d'une absence de } \\
\text { reconnaissance }\end{array}$ & $\begin{array}{l}8 \text { praticiens }(6 \\
\text { hommes et } 2 \\
\text { femmes) }\end{array}$ & $\begin{array}{l}\text { Entretiens } \\
\text { enregistrés. } \\
\text { Temps de } \\
\text { passation en } \\
\text { moyenne de } 80 \\
\text { minutes. }\end{array}$ & $\begin{array}{l}\text { Se référer aux } \\
\text { pages } 103 \text { à } 107\end{array}$ \\
\hline
\end{tabular}

Tableau 2: Récapitulatif de la méthodologie de la recherche

Table 2: Summary of research methodology.

\section{4.- Résultats}

\section{1- Les soins de conservation : les tâches à effectuer}

Ci-dessous, le tableau hiérarchise chronologiquement les tâches à effectuer et les procédés techniques utilisés lors des soins. Ces résultats ne proviennent pas de l'analyse du cadre juridique, mais sont extraits de l'analyse des invariants en phase 1 (phase d'observations). Les procédés techniques détaillent la manipulation d'instruments chirurgicaux (ex: scalpels, trocarts) plaçant les thanatopracteurs en contact direct avec les corps des défunts et d'éléments anatomiques (en particulier le sang). Rappelons que la législation concernant les déchets de soins à risques infectieux (DASRI) souligne l'existence de risques (infectieux ou non), issus des soins de conservation (Articles R.13351; R.1335-2; R.1335-5 à R.1335-8 du code de la santé publique, Collectif, 2004, Charbotel, et al., 
2003, Guez-Chailloux, Puymérail, \& Le Bâcle, 2005).

\begin{tabular}{|c|c|}
\hline Procédés & Temps [min] \\
\hline $\begin{array}{l}\text { Nettoyer complètement le défunt pour obtenir une asepsie externe du corps en vue } \\
\text { d'éliminer les germes et bactéries }\end{array}$ & \multirow[t]{2}{*}{10} \\
\hline Casser les rigidités cadavériques & \\
\hline Vérifier la présence éventuelle d'un pacemaker, puis l'extraire & \multirow{5}{*}{50} \\
\hline $\begin{array}{l}\text { Mettre en place un système composé de pompes manuelles, reliées directement à deux } \\
\text { bocaux. Un des deux bocaux permet la récupération des liquides physiologiques, le } \\
\text { second contient le fluide de conservation (environ } 4-5 \text { litres) qui sera injecté. Le but est } \\
\text { de préserver le défunt de quelques jours à quelques semaines, et contribuer à atténuer } \\
\text { les traces de souffrances ou marques laissées par un grave accident sur le visage }\end{array}$ & \\
\hline $\begin{array}{l}\text { Inciser près du nombril pour éliminer les liquides excédents et le gaz contenu dans } \\
\text { cavités et organes. Les gaz intestinaux sont évacués par une pression manuelle } \\
\text { abdominale }\end{array}$ & \\
\hline $\begin{array}{l}\text { Par l'intermédiaire d'un trocart", le sang est aspiré directement du ventricule droit, } \\
\text { ainsi que les fluides des cavités abdominale et thoracique tels que le surplus de sang, } \\
\text { de liquide pleural, l'urine ou le contenu de l'estomac, avant une seconde injection } \\
\text { d'un produit plus concentré en formol: le formaldéhyde concentré. Cette opération a } \\
\text { pour objectif de retarder et de ralentir la prolifération des bactéries contenues dans les } \\
\text { cavités thoraciques et abdominales. Ces dernières sont les principales causes d'une } \\
\text { évolution effrénée de la putréfaction, constituant un risque majeur en terme de santé } \\
\text { publique, quand elles sont associées à une température élevée. } \\
\text { * Tube de métal allongé avec un embout, relié à un système d'aspiration introduit par } \\
\text { voie abdominale }\end{array}$ & \\
\hline Suturer les incisions & \\
\hline Poser des leurres (couvre-œil et formes bouche) & \multirow{3}{*}{30} \\
\hline $\begin{array}{l}\text { Méchage du nez et de la bouche (rarement l'ensemble des orifices) avec du coton } \\
\text { hydrophile, puis ligaturer la bouche. }\end{array}$ & \\
\hline $\begin{array}{l}\text { Habiller, maquiller, coiffer, présenter (posture, éclairage tamisé, présentation du lit, } \\
\text { etc.) et fixer à la cheville le flacon contenant un échantillon du liquide employé. }\end{array}$ & \\
\hline
\end{tabular}

Tableau 3: Description des tâches à effectuer lors d'un soin

Table 3: Description of tasks to be carried out during a procedure.

\section{2.- Une santé menacée?}

\section{Les maux de dos et les piqûres percutanées}

L'état de santé des praticiens est menacé par des risques biologiques, infectieux, toxicologiques et principalement de pathologies rachidiennes, qui découlent des conditions de travail $(t=.82 ; d l=6$; $p=.0138) .50 \%$ des praticiens interrogés, quel que soit l'âge, estiment « tout le temps » souffrir du dos. L'échantillon interrogé indique que ces conditions de travail concernent des interventions particulières : $100 \%$ déclarent avoir déjà travaillé dans un couloir, 75\% dans un cagibi, 62,5\% sur des fauteuils ou par terre, $50 \%$ dans une cave, et 37,5\% dans une cabane de jardin. D'autre part, $50 \%$ de ces professionnels relatent s'être piqués avec du matériel à plusieurs reprises, voire très souvent: «Se piquer avec du matériel usagé? Oui cela arrive régulièrement, assez souvent dans des moments de fatigue ». 


\section{Les relations professionnelles, les projets professionnels}

Est abordée dans cette recherche la fragilité d'une identité professionnelle de ce corps de métier. $50 \%$ de l'échantillon interrogé n'évoquent jamais leur profession en « société », tandis que $25 \%$ prétendent exercer un autre métier: «Ma famille a très mal réagi à l'annonce de mon métier [...] il a fallu près d'une année. J'ai encore des petites remarques, c'est tourné sur le temps de la plaisanterie, mais je fais quand même attention à mes dires. » $62,5 \%$ des praticiens interrogés déclarent monter des projets dans un autre secteur d'activité. L'ensemble des résultats de cette recherche pointe l'existence d'un besoin marqué de cohésion au sein de ce corps de professionnels. Au risque, pour les praticiens, d'envisager un changement de métier, qui serait initié en raison de différences fondamentales dans l'exercice du métier $(t=.71 ; d l=3 ; p=.0460)$ : «Les motivations des gens sont aussi différentes pour aborder ce métier et cela peut effectivement poser des problèmes. Éthiquement, chacun porte ce métier très différemment, avec sa vision. Ceux de ma génération de diplômés, sont jeunes, sont motivés pour ce qu'ils font ou pour l'argent, [...] c'est un milieu très masculin, les anciens [...] ont parfois plus de mal à accepter qu'un jeune vienne là et soit motivé, cela les dépasse ». On constate une faible relation linéaire entre les attitudes de la sphère sociale/familiale et l'existence de projets professionnels dans un autre secteur d'activité. A contrario, l'attitude des pairs $(t=.71 ; d l=$ $3 ; p=.0460$ ) peut engendrer une démotivation (professionnelle) et à terme, un abandon du métier.

\section{3.- Les soins de conservations : une situation de travail aux nombreuses facettes}

Les résultats présentés dans cette partie sont issus des phases 2 et 3 de la méthodologie.

\section{Une gestion complexe du temps}

Les thanatopracteurs prennent connaissance des soins à effectuer la veille ou le jour même. Planifier à long ou moyen terme est impossible. Ils prévoient un temps de travail de 7 heures à 18 heures, dans lequel les soins seront intégrés. Un second aspect temporel intervient lors de l'exécution des soins. Les thanatopracteurs n'ont peu, sinon jamais, d'informations sur la cause du décès, la morphologie ou l'âge de la personne décédée. Ainsi, les professionnels n'évaluent le type de soins à effectuer et le temps de leur réalisation, que lors de leur arrivée sur les lieux. Divers facteurs tels que la cause des décès, les soins post-autopsie, la morphologie ou l'âge des défunts peuvent prolonger la durée habituelle des soins (1 h 30$)$ de plusieurs heures, renversant l'organisation des soins et contraignant les chambres mortuaires ou funéraires à ne pas se conformer aux horaires de fermetures.

\section{La gestion de l'espace}

La gestion de l'espace se fait au travers des déplacements nécessaires, sur plusieurs départements, pour se rendre sur les lieux des soins. En moyenne, les déplacements quotidiens recouvrent 4 heures du temps de travail. Dans cette recherche, $45 \%$ des soins ont eu lieu dans une chambre mortuaire, $30 \%$ dans une chambre funéraire, $14 \%$ dans un domicile, $10 \%$ dans une maison de retraite et $1 \%$ dans un institut médico-légal. Les disparités de ces différents lieux d'intervention accentuent les contraintes des soins et augmente la charge de travail physique, cognitive et psychique des praticiens $(t=.55 ; d l=4 ; p=.0021):$ "Je fais beaucoup plus attention dans les domiciles, ne pas laisser de traces, ne pas salir, et le résultat doit être immédiat, on doit faire beaucoup plus attention au résultat parce que la réaction de la famille, on l'a tout de suite. Dans un funérarium ou un hôpital, on a rarement de réaction puisqu'il n'y a personne. Les soins à domicile demandent un travail beaucoup plus intense, il faut faire très attention, donc c'est beaucoup plus long, plus fatiguant, il faut être attentif dans ses gestes. Le travail est psychiquement plus dur, je ressors toujours plus vidé, totalement vidé d'un domicile, je suis complètement cuit ». 


\section{Un cadre relationnel}

L'aspect relationnel est une dimension majeure dans l'activité de la thanatopraxie (Caroly, Rocchi, Trompette, \& Vinck, 2003). Les personnels d'accueil connaissent généralement les thanatopracteurs qui interviennent dans leur établissement. Leurs rapports relationnels, majoritairement complaisants, avec ces praticiens influencent la perception des conditions de travail $(t=.54 ; d l=4 ; p=.0194)$. Cependant, dans $50 \%$ des interventions, on note des va-et-vient intempestifs d'un personnel d'accueil désigné comme désagréable, très intrusif et/ou peu ou pas coopératif. Ces va-et-vient présentent un lien significatif avec l'évaluation des conditions travail $(t=.44 ; d l=4 ; p=.0002)$. Les attitudes intrusives ou d'évitement du personnel d'accueil sont perçues par les praticiens comme discriminatoires et de rejet, et les va-et-vient comme une surveillance abusive: "J'évite que les gens qui passent puissent voir ce que je fais. [...] Des fois, il y en a certains qui cherchent n'importe quel prétexte pour pouvoir regarder ce qu'on fait. [...] Dans les domiciles, il faut toujours surveiller la porte ».

L'absence de personnel d'accueil lors des soins de conservation dans des établissements peut aussi apparaître comme un facteur contraignant: "En l'absence de matériel [...], on ne se déplace pas pour demander [...]. Non. Soit on se débrouille seul, soit on ne demande rien, [...] plus vous demandez, plus vous allez vous attirer des ennuis ». Il subsiste ainsi une notion (d'absence) de territoires de compétences (Caroly et al., 2003) à l'origine de frictions. Par ailleurs, certains soins, qui nécessitent plusieurs heures, peuvent entraver l'organisation interne de ces établissements (horaires de fermeture), ce qui n'est pas sans conséquence sur les horaires des personnels d'accueil (Caroly, et al., op. cit.).

\section{Les aléas des soins}

Les soins se déroulent selon une chronologie précise, mais varient selon certains facteurs (facilités techniques, fonctionnalité des locaux, caractéristiques liées au défunt). Les facilités techniques des thanatopracteurs se composent de tables opératoires réglables ou lits médicalisés, d'appuie-têtes, d'éviers avec arrivée d'eau, de poubelles D.A.S.R.I., de plans de travail, de produits bactéricides et d'essuie-mains. Les locaux adéquats comportent un espace de travail fermé, un système d'aération et de renouvellement de l'air, un éclairage puissant, une asepsie parfaite (Monsembernard, \& Vidal, 2003).

Ces équipements sont peu présents dans les situations réelles de travail. Pourtant, un lien est établi entre ces éléments et l'évaluation de la pénibilité des conditions de travail $(\mathrm{p}<.0001)$. Dans 40 à $52 \%$ des interventions, on relève leur absence, à l'origine de nombreuses stratégies palliatives et catachrèses (ex: utilisation d'un rouleau de papier toilette en guise d'appui-tête, gels antibactériens en cas d'absence d'eau).

a) Les ambiances visuelles, qui nécessiteraient un éclairage chirurgical ou « sans ombre » (de 750 à 1000 lux) au poste de travail, se composent généralement d'un éclairage unique, direct et artificiel (plafonnier inférieur à 500 lux). L'éclairage tient un rôle majeur lors des interventions $(t=.58 ; d l=$ $4 ; p<.0001$ ), les praticiens ne peuvent assurer correctement la technicité des soins (incisions, prélèvements), la phase de présentation (nuancer les maquillages), et éviter les risques professionnels (piqûre percutanée, émanations du formol et gaz) liés aux erreurs de manipulations: "L'éclairage est généralement très mauvais et, la source, est toujours décalée. C'est un problème pour les soins, on peut avoir une mauvaise vision de ce que l'on fait, et pour la présentation, ça peut fausser au niveau des couleurs.»

b) Les hauteurs de plateaux non réglables ou lits non médicalisés imposent d'adopter des postures extrêmes de travail (ex: génuflexions, rotations du torse à $\left.45^{\circ}\right)(t=.69 ; d l=4 ; p=.0005) .62,5 \%$ des évaluations « très pénibles » des conditions de travail notent l'utilisation de « lits trop bas » et les tables réfrigérantes servent de tables opératoires pour les soins. De même, l'absence ou l'inadéquation des plans de travail et/ou tablettes pour disposer les instruments contraint les professionnels $(t=$ $.52 ; d l=4 ; p<.0001$ ) dans leurs gestes professionnels en disposant les instruments hors de portée: «On n'a jamais de paillasse pour installer notre matériel, je prends une petite boite Tupperware 
dans laquelle je les installe. Autrement, je suis obligé de les laisser dans la valise des instruments que je pose généralement par terre, ce qui fait que je travaille très souvent par terre. »

c) Pourtant nécessaire afin de renouveler l'air vicié présent dans les salles de soins fermées (normes $\pm 30 / 45 \mathrm{~m}^{3} /$ heure/occupant $)$, l'absence d'aération contribue à dégrader les ambiances de travail $(t=$ $.61 ; d l=4 ; p<.0001$ ) chargées d'émanations de formol et odeurs dites nauséeuses: «Les yeux, les muqueuses nasales et la peau du visage sont souvent irrités par les produits chimiques. On peut aussi se prendre des coups de formol, se prendre des bouffées de formol par mauvaise manipulation et cela se traduit par des migraines qui durent deux à trois jours sans discontinuer ».

d) Concernant la prise en charge des D.A.S.R.I., l'absence de conteneurs adaptés influence le cadre de travail $(t=.65 ; d l=4 ; p<.0001)$ et oblige les praticiens à mettre en place, par leurs propres moyens, un système de récupération des déchets des soins et de les éliminer: "Les poubelles, elles, sont souvent pleines, elles débordent. On ne dispose pas toujours de conteneurs pour les déchets de soins, parfois il n'y a pas de poubelles, tout court. J'ai toujours des rouleaux de sacs poubelle sur moi [...] un sac plastique [...] puis je le ferme, je l'emmène avec moi et je le jetterai un peu plus tard dans un hôpital ou une poubelle».

Ces éléments matériels sont essentiels aux conditions de travail, à la maîtrise des risques et à la préservation de la santé des thanatopracteurs. Or, $75 \%$ des évaluations des conditions de travail à la suite d'un soin et déclarées « très pénibles » notent l'absence de l'ensemble de ces éléments. Indépendamment du niveau de pénibilité estimée, un tiers de l'ensemble des interventions note l'absence a minima d'un élément et une hygiène insuffisante des locaux. Ces matériels, indispensables à cette activité et consécutivement à la santé des praticiens, pourraient paradoxalement être aisément mises en place dans les établissements médicalisés et/ou spécialisés.

\section{Une dimension émotionnelle}

L'aspect émotionnel à l'origine d'une dénégation occupe une place essentielle. L'habitude est un moyen de minorer la dureté latente du métier, mais les praticiens déclarent toujours considérés les « individus » qu'ils traitent. Des situations de travail peuvent être douloureuses, voire impossibles pour certains (ex: mineurs, morts violentes). De plus, travailler seul est une caractéristique dans cette profession. Cette solitude, appréciée la plupart du temps, peut cependant être mal vécue dans les contextes relationnels conflictuels (ex: soins à domicile très redoutés). Les praticiens interviennent juste après un décès, ce qui correspond à une période très angoissante pour les proches (Cornillot, $\&$ Hanus, 2000) pouvant percevoir la venue des thanatopracteurs comme particulièrement intrusive.

En cas d'obligations législatives d'effectuer des soins, les thanatopracteurs peuvent être contraints de repartir des domiciles sans avoir effectué les soins parfois perçus comme une atteinte grave à la personne décédée.

Les professionnels rapportent des situations si conflictuelles, qu'elles aboutissent à des menaces verbales et des passages à l'acte. De tels conflits peuvent survenir avec les toiletteurs rituels ${ }^{4}$. La présence de ces intervenants et l'évaluation des conditions de travail ont une corrélation significative $(t=.42 ; d l=4 ; p=.0197)$. Un espace de travail insuffisant et un climat conflictuel, contribuent, pour les praticiens, dans 66,67\% des observations à estimer les conditions de travail comme «pénibles »: «C'est toujours délicat avec les intervenants extérieurs, les toiletteurs rituels ne nous aiment pas trop et le font savoir. C'est arrivé qu'on me menace ». Les divers constats ressortis de cette recherche renvoient aux problématiques similaires rencontrées par les thanatopracteurs avec les personnels d'accueil (cf. le cadre relationnel) et témoignent d'une absence de délimitation des territoires de compétence, et d'un malaise social, générés par l'objet même de cette activité.

4. Les toiletteurs ont pour fonction de laver et préparer les corps des personnes décédées selon un rituel (bouddhisme, islam, judaïsme). 


\section{5.- Discussion}

Le processus de rationalisation de la pensée occidentale relative à la mort ôte le contenu moral et le sacré religieux pour, en définitive, ne considérer que la finalité et les moyens sélectionnés (Weber, 2006). Ce phénomène progressiste, et pourtant réducteur, apparaît à l'origine d'une tabouisation de la mort et par extension de la thanatopraxie. L'utilisation et le rapport au corps désormais méprisés (Rizet, 1998) et les expériences négatives du mépris rencontrées par les praticiens conduisent à une aliénation sociale (Sigaut, 1991), porteuse d'une lutte pour la reconnaissance de l'identité lésée des thanatopracteurs (Honneth, 1996). Les dimensions relationnelle et émotionnelle de cette activité comprennent des stratégies de dissimulation de la profession exercée (omission, mensonge) par $75 \%$ des professionnels interrogés, mais aussi des comportements d'évitement face à un personnel d'accueil peu et pas coopératif, et des activités empêchées lors par exemple de refus de partage (par les toiletteurs rituels) des lieux d'interventions. Témoins d'une souffrance au travail, les abandons de métier évoqués et envisagés par les professionnels ou supposés (rapport praticiens/nombre de diplômés) reflètent très justement l'existence d'activités « empêchées » (Clot, 2002). Ces situations s'analysent dans leur dimension contextuelle, car c'est bien sur le métier (ou la profession) qu'il faut soigner et ce sur quoi il faut agir (Clot, \& Faïta, 2000).

L'état actuel du contexte juridique de la thanatopraxie française reflète la subordination historique de la profession au statut monopolistique du secteur funéraire, octroyé aux pompes funèbres. Cette subordination peut s'avérer dramatique notamment sur les plans émotionnel et relationnel. Ne sont plus comptés, les insultes, les menaces et les passages à l'acte envers les thanatopracteurs, engendrés suite à des ventes abusives ou détournées de soins, par les pompes funèbres. Deux constats majeurs émanent de l'analyse de ce contexte juridique. Tout d'abord, et à l'image de la caractéristique ambulatoire, les « carences » d'un cadre réglementé qui se traduisent par l'absence d'équipements et de locaux qui intégreraient la gestion complexe du temps, de l'espace, du cadre relationnel et des aléas des soins (Caroly et al, 2003). Ensuite, les rares applications et respect des conditions édictées: les conditions de travail et les facilités techniques sont systématiquement indéterminées. Les cahiers des charges des lieux des soins ne répondent pas aux impératifs (exception faite des funérariums et chambres mortuaires récentes) en ne procurant ni les équipements (ex : poubelles D.A.S.R.I) ni les installations (ex: aération conforme, espace au sol) requises. Quant aux domiciles, ils n'ont, bien entendu, aucune possibilité d'accueillir les thanatopracteurs.

Révélateur d'une absence de définition des territoires de compétences (Caroly et al., op. cit.), ce statut requiert pourtant un diplôme (depuis 1994) et est assujetti à des obligations législatives de faire effectuer ou de proscrire les soins. Une mission (licence) - celle de la prise en charge d'un tabou -, est confiée à ces professionnels par l'Etat, qui, en revanche, exclut et ne concède ni le sens social, ni le contrôle de l'exercice du métier (mandate) (Hughes, 1996). Cela témoigne d'éléments défaillants à la constitution d'une profession, à la fois explicatifs et générateurs des caractéristiques de cette activité (ex: dimension ambulatoire) et des conditions d'exercice pénibles, voire dangereuses, à l'image de l'état de santé menacée des praticiens.

Tout métier a certes une part de « sale boulot » (Hughes, op. cit.), mais la thanatopraxie en deviendrait moins une activité comprenant une part de « sale boulot », qu'un « sale boulot », un « dirty work » lui-même. Les professionnels souffrent de la représentation des objets « souillés » qu'ils manipulent et des comportements « impurs » et « choquant » qu'ils adoptent, mais à laquelle ils opposent le rôle de l'acte rétroactif (par le leurre) de leurs soins. Faute de quoi, ces praticiens ne se résumeraient qu'à collecter, traiter et participer à l'enlèvement d'objets (cadavres), tels des éboueurs, des rippers de morts. Dans l'analyse de stratégies défensives du travail, des liens ont été établis entre des activités relatives à la mort et aux déchets (Weiss, 1993). Ces activités prennent en charge (stockage, élimination, transport, etc.) au terme d'un processus (ex: soins palliatifs, collecte des déchets, etc.) les objets n'ayant plus de raison d'être. Irrémédiablement peu valorisées, à l'image des éboueurs, elles bénéfi- 
cient d'une faible reconnaissance et de conditions de travail à la pénibilité marquée (Volkoff, 2006).

\section{Conclusion}

La profession des thanatopracteurs représente une « rubrique qui ne comporte pas d'inscrit $»^{5}$, une composante juridique partielle et une identité fragilisée. Elle subsiste grâce aux opérateurs qui élaborent leurs propres règles, leurs styles personnels (Clot, 2002). Cependant, l'empêchement de cette construction collective étiole inexorablement le genre professionnel et les nombreux abandons de métier témoignent de cette souffrance au travail (Clot, \& Faïta, 2000).

Une des pistes de transformation reposerait sur l'observation et l'analyse du modèle congru de la thanatopraxie nord-américaine. Cette dernière, à l'inverse de la pratique française et de sa caractéristique ambulatoire, impose que les corps des défunts soient amenés auprès des praticiens. Les thanatopracteurs interviennent ainsi dans un cadre et des conditions de travail maîtrisées et contrôlées. Ce système contribue à garantir l'autonomie des professionnels, à protéger les praticiens et à assurer aux familles un déroulement optimal des soins de conservation. Notons que la thanatopraxie nordaméricaine apparaît comme une profession reconnue, respectée et monopolistique. Les objectifs futurs nécessiteraient de promouvoir des réflexions sur la constitution d'un corps de métier cohérent et solidaire, d'examiner, au plus près, les conditions de travail des thanatopracteurs français et de les légitimer d'un point de vue juridique. À cet égard, il semblerait inopportun de considérer que les ergonomes ne puissent pas intervenir au-delà du champ même du travail. Dans la situation exprimée ici, cette dernière et sa problématique ne peut, selon nous, être pleinement saisie, comprise, et par conséquent transformée sous le seul prisme de l'activité. Or, seule, l'intervention des ergonomes a permis de mettre en lumière les conditions de travail et les impacts sur les opérateurs. Alors, quelle(s) marge(s) de manœuvre et quel(s) objectif(s) pour l'ergonome une fois le travail « compris » (Biotteau \& Mayeur, 2008) ? Au-delà d'une transformation du travail, il s'agit ici, de saisir la complexité des réalités et logiques dans lesquelles s'inscrivent cette profession, mais aussi les professions, afin d'œuvrer à une transformation qui dépasse le simple cadre du travail et qui agisse, au sein d'une réflexion transdisciplinaire, sur les interactions d'une systémie sociétale. Ainsi pourrait-on voir dans la posture de cette écologie humaine, de cette transdisciplinarité, une nouvelle forme de contribution à visée sociétale de l'ergonomie.

\section{RÉFÉRENCES}

Aguilly, F., Batisse-Dauquaire, P., Bonello, L., Bremaud, M.M., Caquineau, J., Dupont, M., France, M., Genyk, I., Grand-Sebille, C., Hauw, J-J., Hirsch, E., Jacquard, T., Laval, F., Noel, J-Y., Rigaud, G., Romiguiere, M., \& Wolf, J. (2004). Soigner après la mort. Pratiques en chambres mortuaires. Paris: Collection Espace éthique/ AP-HP, Hors série/3, 50 p.

Alby, M-L., Benattar, L., Calvat, T., Caquet, R., Chapuisat, X., Charpentier, B., Causse, D., Dascenso, D., Demarest, M., Depecker, L., Desaulle, M-S., Elenberg, E., Faugeres, J-P., Fiat, E., Gagey, O., Guichardon, M., Hardy, P., Hemon, D., Hervy, M-P., Hirch, E., Joublin, H., Jougla, E., Julien-Laferriere, F., Koskas, A., Laroque, G., Leduc, F., Ladrans, M., Moulias, R., Ollivet, C., Pellestier, J., Sebag-Lanoë, R., Spira, A., Svandra, P., \& Van Lerberghe, R-M. (2004). Vieillesses méconnues. Enjeux éthiques de la crise d'août 2003. In E. Hirsch (Ed.), 6 ${ }^{e m e}$ colloque d'éthique de Bicêtre. Lassay-les-Châteaux: Collection Espace éthique/ AP-HP, $106 \mathrm{p}$.

Anonyme. (1992). Catéchisme de l'église catholique. Poitiers: Mame/ Plon, 676 p.

Ariès, P. (1975). Essai sur l'histoire de la mort en occident du moyen âge à nos jours. Paris: Seuil, collection Points, $237 \mathrm{p}$.

Bacqué, M-F. (2002). Vers une mondialisation des rites funéraires? L'Esprit du temps, Etudes sur la mort, 2002/121, 85-95.

5. Résultat d'une recherche du nom « thanatopracteur » dans les pages jaunes. 
Biotteau, M., \& Mayeur, A. (2008). Thanatopracteurs : «Cette rubrique ne comporte pas d'inscrits » Quelles transformations du travail pour une activité sans métier défini? In P. Negroni, \& Y. Haradji (Eds.), Ergonomie \& Conception. "Concevoir pour l'activité humaine » [43ème congrès de la SELF, Ajaccio, France, 17-19 septembre 2008] (pp. 127-134). Ed. ANACT.

Caroly, S., Rocchi, V., Trompette, P., \& Vinck, D. (2003). Les services au défunt. Acteurs, territoires de compétences et dynamiques professionnels. Rapport de recherche, convention Mi- DRESS, $\mathrm{n}^{\circ} 22 / 01$, Centre de recherche innovation sociotechnique et organisations industrielles. Université Pierre-Mendès France, non publié, 1-4, 34-5.

Charbotel, B., Forissier, M.F., Hours, M., \& Bergeret, A. (2003). Revue sur les risques professionnels liés à l'élimination des déchets d'activité de soins. Archives des maladies professionnelles et de médecine du travail, 64(2), 100-105.

Clot, Y. (2002). La fonction psychologique du travail (Troisième édition augmentée). Paris: PUF.

Clot, Y., \& Faïta, D. (2000). Genres et styles en analyses du travail. Concepts et méthodes. Travailler, $\mathrm{n}^{\circ} 6$.

Collectif (2003). Code général des collectivités territoriales (CGCT). Paris: Dalloz, coll. Codes Dalloz, $6{ }^{\text {ème }}$ édition, 158, 159, 785-797.

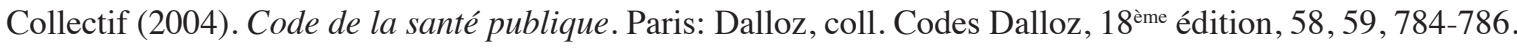

Cornillot, P., \& Hanus, M. (2000). Parlons de la Mort et du Deuil. Bonchamp-Lès-Laval: Frison Roche (3 3 ème édition), 31-75.

Dant, T., \& Bowles, D. (2003). Dealing with Dirt: Servicing and Repairing Cars. Sociological Research Online, 8(2). http://www.socresonline.org.uk/8/2/dant.html.

Desesquelles, A., \& Richet-Mastain, L. (2004). Bilan démographique 2003 : stabilité des naissances, augmentation des décès. INSEE première, $\mathrm{n}^{\circ} 948$.

Douglas, M. (1984). Purity and Danger: An Analysis of the Concepts of Pollution and Taboo. London: Routledge (1ère édition), 272 p.

Freidson, E. (1971). Profession of Medicine: A Study of the Sociology of Applied Knowledge. New York: Dodd, Mead, 208 p.

Frémy, D., \& Frémy, M. (2003). Le Quid 2004. Malesherbes: Robert Laffont, 633, 1599.

Gauchet, M. (1985). Le désenchantement du monde. Une histoire politique de la religion. Paris: Gallimard.

Guez-Chailloux, M., Puymérail, P., \& Le Bâcle, C. (2005). La thanatopraxie: état des pratiques et risques professionnels. INRS, Documents pour le médecin du travail, $\mathrm{n}^{\circ}$ 104, $4^{\mathrm{ème}}$ trimestre 2005, 451-469.

Guibe-Hubert, N. (2002). Présentation des divers métiers du funéraire et de leurs risques professionnels. Rapport d'étude de la société médecine du travail Normandie (SMTN), non publié, 10 p.

Honneth, A. (1996). La dynamique du mépris. D’où parle une théorie critique de la société ? In Ch. Bouchindhomme, \& R. Rochlitz (Eds.), Habermas, la raison, la critique (pp. 216-238). Paris: Les Editions du Cerf, coll. « Procope».

Hughes, E. C. (1996). Le regard sociologique : essais choisis. Paris: Éditions de l'École des hautes études en sciences sociales.

Jean, P. (2006). Synthèse du chapitre IX: La reconnaissance du travail, «Les enjeux psychiques du travail » de Pascale Molinier, non publié, 2 p.

Mayer, R. G. (2000). Embalming: History, Theory and Practice. West Ford: Sally J. Barhydt, 3 ème édition, 4.

Monsembernard, G., \& Vidal, R. (2003) Guide pratique de législation funéraire. Paris: Juris Classeur Litec, coll. Guides pratiques, $304 \mathrm{p}$.

Pla, A. (2008). Bilan démographique 2008 - Plus d'enfants, de plus en plus tard. INSEE première, $\mathrm{n}^{\circ} 1220$. http://www.insee.fr/fr/ffc/ipweb/ip1220/ip1220.pdf 
Poitou, J.-P. (1992). Nouvelles technologies et élévation des qualifications : à propos du rôle de la visuomotricité et de la motricité graphique dans l'activité cognitive globale du technicien de bureau d'étude. Intellectica, 1/2(13-14), 185-217.

Prioux, F. (2006). L'évolution démographique récente en France. Population 2006/4, Volume 61, 393-435.

Rizet, C. (1998). Aux sources d'une culture du mépris. Le journal des psychologues, 160, 14-17.

Robert-Bobée, I. (2006). Projections de population pour la France métropolitaine à l'horizon 2050. INSEE première, $n^{\circ} 1089$. http://www.insee.fr/fr/ffc/ipweb/ip1089/ip1089.html

Sigaut, F. (1991). L’Animal, machine ou personne? Ethnozootechnie, 46, 5-12.

Thomas, L.-V. (1980). Anthropologie de la mort. Paris: Payot.

Thomas, L.-V. (2003). La Mort. Paris: PUF, Collection Que sais-je ?, n² 236, 128 p.

Volkoff, S. (2006). « Montrer » la pénibilité: le parcours professionnel des éboueurs. In G. Balazs, M. Gollac, \& S. Volkoff. (Eds.), Santé et travail. Déni, visibilité, mesure. Actes de la recherche en sciences sociales, $\mathrm{n}^{\circ} 163$, juin, pp. 62-71.

Weber, M. (2006). Sociologie de la religion. Paris: Flammarion, 512 p.

Weiss, M. (1993). Bedside manners: Paradoxes of physician behavior in grand rounds. Cultural, medecine and psychiatry, 17(2), 235-253.

\section{RÉSUMÉ}

Cette recherche ergonomique décrit les invariants et la variabilité des situations de travail des thanatopracteurs. Elle s'interroge quant aux difficultés relatives aux conditions de travail posées et à leurs impacts en matière de santé sur les praticiens. Cependant, la complexité que revêt cette profession, qu'il est essentiel de saisir pour la compréhension du travail et sa transformation, nécessite une approche transdisciplinaire, et ce, afin de distinguer les différents niveaux de réalité et de logique sous-jacents. La thanatopraxie apparaît indispensable à la survivance d'une société, qui pourtant, la qualifie et la considère de dégradante. Cette profession qui consiste à prendre en charge des « objets » « sales »: les cadavres, illustration du tabou de la mort, n'est souhaitée par personne. Nous verrons comment cette profession, du point de vue sociétal, peut apparaître comme un «sale boulot » duquel découlerait un contexte juridique parcellaire à l'origine d'une aliénation sociale et d'un cadre de travail pathogène.

\section{MoTS CLÉS}

Thanatopracteurs, tabou, « sale boulot », contexte juridique, santé, conditions de travail.

\section{RÉFÉRENCEMENT}

Biotteau, M., \& Mayeur, A. (2010). La prise en charge du tabou de la mort par les thanatopracteurs: conséquences sur la santé et les conditions de travail. Activités, 7(1), pp. 95-110. http://www.activites.org/ v7n1/v7n1.pdf

Article soumis le 3 mai 2009, accepté pour publication le 15 février 2010 